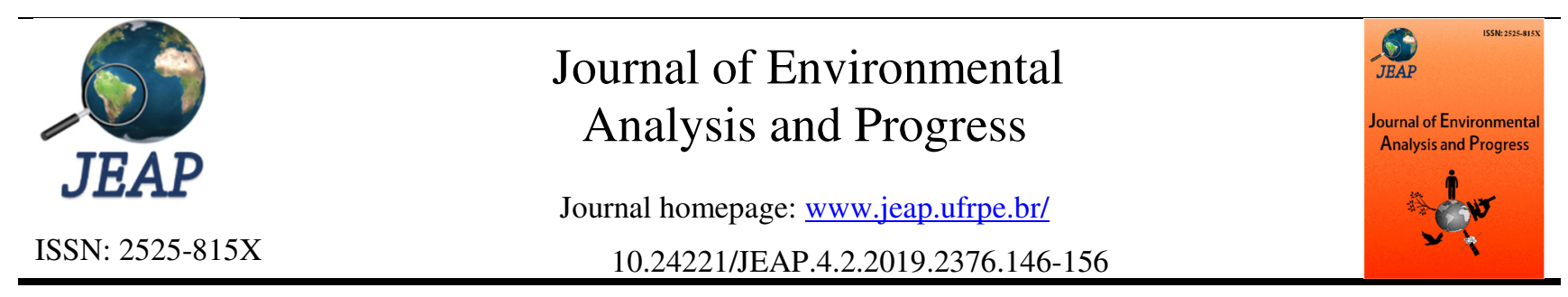

\title{
O efeito do método de desagregação de chuva no hidrograma de projeto para uma bacia hidrográfica rural no semiárido nordestino
}

\section{The effect of the method of disaggregation of rain in the hydrograph project for a rural hydrographic basin in the northeastern semi-arid region}

Artur Paiva Coutinho ${ }^{\mathrm{a}}$, Lucas Ravellys Pyrrho de Alcântara ${ }^{\mathrm{b}}$, Ialy Rayane de Aguiar Costa ${ }^{\mathrm{c}}$, Vitor Hugo de Oliveira Barros ${ }^{\mathrm{d}}$, Larissa Fernandes Costa ${ }^{\mathrm{b}}$, Larissa Virgínia da Silva Ribas ${ }^{\mathrm{a}}$, Edevaldo Miguel Alves ${ }^{\mathrm{a}}$, Antônio Celso Dantas Antonino ${ }^{\mathrm{b}}$

a. Universidade Federal de Pernambuco-UFPE, Centro Acadêmico do Agreste. E-mail: arthur.coutinho@yahoo.com.br, larissa.vribas@hotmail.com, edevaldofisica@gmail.com.

b. UFPE, Departamento de Energia Nuclear-DEN, Programa de Pós-Graduação em Tecnologias Energéticas e Nucleares-PROTEN. Av. Luiz Freire, n. 1000. Cidade Universitária, Recife-PE, Brasil. CEP: 50740-545. E-mail: ravellyspyrrho@gmail.com, larissafercosta@ hotmail.com, acdantonino@gmail.com.

c. UFPE, Programa de Pós-Graduação em Engenharia Civil. E-mail: ialy_rayane@ hotmail.com.

d. UFPE, Programa de Pós-Graduação em Engenharia Civil e Ambiental. E-mail: vitor_barros1 @ outlook.com.

A R T I C L E I N F O

Aceito 30 Abr 2019

Publicado 30 Abr 2019

\begin{abstract}
A B S T R A C T
The evaluation of the flow of projects of extreme importance for the design of several works of hydraulic strategy. Considering this context, this study aims evaluate the influence of the methodology of Bell and the relations between the duration in the disaggregation of maximum daily precipitations to obtain the hydrograms of the project. A study basin was built for Riacho da Cachoeira, located in the state of Bahia, and as a set of historical data applied by the National Water Agency. For the construction of the study hietograms, the methods of the Complaints Office and the Alternate Blocks were used. For the construction of the soil conservation system, the synthetic unit hydrocarbon methodology was used. The Gumbel distribution was able to represent the region at the significance level of $5 \%$. The method of relations between durations had a coefficient of determination of $99.79 \%$ while the method of Bell obtained a coefficient of $99.64 \%$. The Bureau of Complaint method generated a higher flow peak than the Alternate Blocks. The Bell method gave rise to larger openings and tensile intensities, which is the ratio of the durations. Bell's method with the Bureau of Reclamation was presented as the main forms of presentation of durations with the Alternate Blocks method presented as minorities.
\end{abstract}

Keywords: Bureau of Reclamation, alternate blocks, Gumbel, Bell, relationships between durations, statistical hydrology.

\section{R E S U M O}

A determinação da vazão de projeto é de extrema importância para o dimensionamento de diversas obras de infraestrutura hidráulica. Nesse contexto, esse estudo objetiva avaliar a influência da metodologia de Bell e das relações entre durações na desagregação de precipitações máximas diárias para a obtenção de hidrogramas de projeto. A bacia de estudo utilizada foi a do Riacho da Cachoeira, localizada no estado da Bahia, e as séries históricas utilizadas foram obtidas na Agência Nacional de Águas. Para a construção dos hietogramas de projeto foram usados os métodos do Bureau of Reclamation e dos Blocos Alternados. Para a construção do hidrograma foi utilizada a metodologia do hidrograma unitário sintético do Soil Conservation Service. A distribuição Gumbel foi capaz de representar as precipitações extremas na região ao nível de significância de 5\%. O método das relações entre durações apresentou um coeficiente de determinação de $99,79 \%$ enquanto que o método de Bell obteve um coeficiente de $99,64 \%$. O 
método do Bureau of Reclamation gerou um pico de vazão maior que o método dos Blocos Alternados. O método de Bell gerou vazões e intensidades de precipitação maiores que o método das relações entre durações. O método de Bell com o Bureau of Reclamation apresentou as maiores vazões e o método das relações entre durações com o método dos Blocos Alternados apresentou as menores vazões.

Palavras-Chave: Bureau of Reclamation, blocos alternados, Gumbel, Bell, relações entre durações, hidrologia estatística.

Introdução
A determinação da vazão de projeto é de
extrema importância para o dimensionamento de
diversas obras de infraestrutura hidráulica. Para a
determinação da vazão máxima provável da bacia,
comumente são utilizados modelos chuva-vazão,
os quais determinam a vazão de projeto a partir de
uma chuva de projeto. Segundo Bemfica,
Goldenfum \& Silveira (2000), os hietogramas são
formas gráficas que demonstram a intensidade da
chuva ao longo de sua duração, eles representam
uma chuva de projeto que serve como entrada ao
modelo chuva-vazão. Portela, Marcos \& Carvalho
(2000) definem hietograma como um acontecimento pluviométrico fornecido por meio de diagramas cronológicos discretos dos sucessivos valores da precipitação.

Os hietogramas podem ser obtidos por meio de equações intensidade - duração frequência (IDF), as quais correlacionam uma intensidade de chuva intensa a uma duração e um tempo de recorrência (tempo de retorno). A determinação das equações de chuvas intensas é de difícil obtenção devido à falta de dados pluviográficos, no entanto metodologias de desagregação de chuvas junto a dados pluviométricos possibilitam determinar os parâmetros das equações IDFs.

$\mathrm{Na}$ literatura, as metodologias de desagregação de chuvas mais utilizadas são a de Bell (1969) e das relações entre durações (CETESB, 1980). Coutinho et al. (2013) utilizaram o método de Bell para estimar os parâmetros das equações de chuvas intensas nas mesorregiões do estado de Pernambuco. Silva \& Oliveira (2017) determinaram parâmetros de chuvas intensas para o nordeste brasileiro utilizando o método das relações entre durações e a função inversa de Gumbel. Garcia et al. (2011) utilizaram os dois métodos comparados com os dados pluviográficos para avaliar a determinação da equação IDF para três estações meteorológicas do Estado de Mato Grosso.

Diversos métodos estão disponíveis para a estimativa de hidrogramas de projeto, no entanto, deve-se respeitar as condições em que eles podem ser aplicados. O Método Racional, método tradicional mais utilizado por diversos autores e projetistas, deve ser utilizado para bacias pequenas, com área de até $4 \mathrm{~km}^{2}$; o Método Racional Corrigido deve ser utilizado para bacias com área entre $4 \mathrm{~km}^{2}$ e $10 \mathrm{~km}^{2}$. Em bacias com área maior que $10 \mathrm{~km}^{2}$ deve ser utilizada a metodologia do Hidrograma Sintético Unitário, conhecida como o método do Soil Conservation Service (SCS).

Comumente utilizado para determinação de hidrograma de projeto, o hidrograma unitário sintético do SCS é um modelo chuva-vazão bastante utilizado por se basear em características fisiográficas da bacia de fácil determinação. Para Carvalho \& Chaudrhy (2001), a facilidade de aplicação do HU encontra-se nas suposições simplificadoras de que a bacia hidrográfica se comporta como um sistema linear e invariante no tempo.

Bemfica, Goldenfum \& Silveira (2000) avaliaram a aplicação de diferentes modelos de hietogramas no método SCS para a criação de hidrogramas unitários. Portela, Marques \& Carvalho (2000) obtiveram os hidrogramas de cheia mediante a aplicação do hidrograma unitário sintético do SCS, com recurso do programa HEC1. Nunes \& Fiori (2007) avaliaram a variação da vazão máxima da bacia hidrográfica do rio Atuba em três períodos distintos de urbanização (a bacia em seu estado natural, início do processo de urbanização e o aumento das regiões urbanas), durante a simulação da vazão máxima foi utilizado o método de Ven Te Chow - Soil Conservation Service (SCS). Mata-Lima et al. (2007) abordaram diferentes métodos de cálculo do tempo de concentração e vazão máxima na bacia hidrográfica da ribeira do Livramento, na região de Setúbal (Portugal), pelo método do SCS.

Damé et al. (2010) compararam os hidrogramas de projeto resultantes dos hietogramas estimados pelas relações IntensidadeDuração-Frequência, obtidos por dados pluviométricos e pluviográficos, para a localidade de Pelotas, RS. Na bacia do ribeirão Serra Azul, Minas Gerais, Cunha et al. (2015) compararam a precipitação efetiva e os hidrogramas de cheia de projeto resultantes da aplicação da metodologia do SCS com eventos de chuva e vazão observados. Drumond, Moura \& Coelho (2018) compararam os dados de monitoramento de um microrreservatório implantado em um empreendimento hospitalar de Belo Horizonte, com os resultados determinados por meio dos métodos SCS e Racional. 
O Riacho Cachoeira é um curso d'água intermitente, ou seja, escoa durante as estações chuvosas e seca na estiagem. Durante as estações chuvosas, o riacho transporta todo o tipo de deflúvio, pois o lençol d'água subterrâneo conserva-se acima do leito fluvial. Este riacho intercepta a estrada municipal que conecta a rodovia estadual BA-210 com o povoado de São Pedro, por este motivo, verifica-se a necessidade da estimativa da vazão máxima, da provável contribuição da bacia do riacho, associada a um determinado tempo de recorrência. $\mathrm{O}$ conhecimento desta vazão de projeto é de suma importância para subsidiar o dimensionamento hidráulico de obras de infraestrutura. Embora as metodologias de desagregação de chuva e de determinação da chuva mais crítica sejam de fácil implementação computacional, poucos estudos avaliam o impacto no hidrograma de projeto em função da escolha do método de degregação de chuva e do método de distribuição mais crítica da precipitação de projeto.
Nesse contexto, esse estudo utiliza como estudo de caso a bacia do Riacho Cachoeira para determinação da vazão máxima provável associada aos períodos de 2, 10, 25, 50 e 100 anos, utilizando IDFs determinadas pelo método de Bell (1969) e o método das relações entre durações.

\section{Material e Métodos}

Área de estudo

A área de estudo compreende a bacia hidrográfica do Riacho da Cachoeira, localizada no município de Sento Sé, Bahia. Foi obtido junto a Superintendência do Desenvolvimento do Nordeste - SUDENE, a carta Serra do Brejinho (Ex - Sento Sé) - Folha SC.24-V-C-V na escala 1:100.000, desenvolvida pelo Ministério do Exército. Com esta carta, foi delimitada a bacia do riacho da Cachoeira, determinando-se a área de contribuição pluvial do riacho até a estrada municipal, que é de aproximadamente $171,17 \mathrm{~km}^{2}$ (Figura 1).

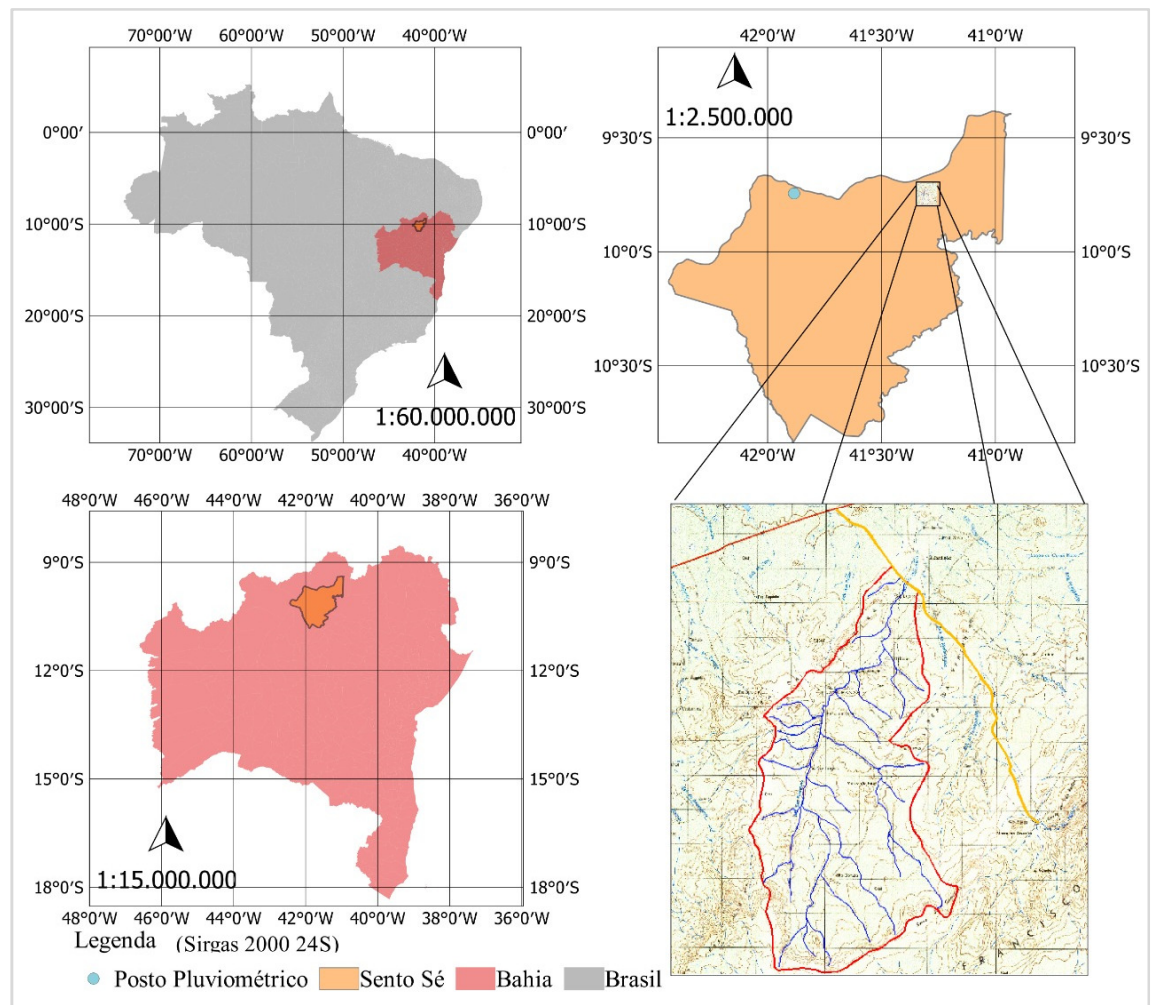

Figura 1. Visão geral da bacia contribuinte delimitada (bacia do riacho da Cachoeira até interseção com a estrada) - sem escala.

\section{Dados pluviométricos}

Foram obtidas séries históricas de diversas estações pluviométricas na região de estudo através da página (web) da Agência Nacional das Águas (ANA, 2012). Além disso, analisaram-se os intervalos disponíveis de monitoramento dos dados e as falhas existentes no período de medição nas séries supracitadas. Como resultado desta análise, foi selecionado o posto Sento Sé, que apresenta 54 anos de dados, como sendo o de melhor representatividade pluviométrica da bacia. $\mathrm{Na}$ Tabela 1 são apresentadas as características do posto utilizado na determinação da precipitação de projeto. 
Tabela 1. Posto utilizado para determinação da precipitação de projeto.

\begin{tabular}{lcccc}
\hline Posto & Código & Bacia & Estado & Município \\
Sento Sé & 941012 & Rio São & Bahia & Sento Sé \\
\hline $\begin{array}{l}\text { Órgão } \\
\text { responsável }\end{array}$ & Latitude & Longitude & $\begin{array}{c}\text { Altitude } \\
(\mathbf{m})\end{array}$ & $\begin{array}{c}\text { Intervalo } \\
\text { de dados }\end{array}$ \\
DNOCS & $-9: 44: 26$ & $-41: 53: 01$ & 380 & $1938-1991$ \\
\hline
\end{tabular}

\section{Tempo de concentração}

Existem vários modelos que estimam o valor do tempo de concentração em função das características da bacia (área, declividade, comprimento do talvegue, rugosidade da superfície, entre outras). Deste modo, deve-se aplicar o modelo que melhor se adeque às características da região. Para bacias rurais como a bacia do Riacho da Cachoeira, o modelo de Kirpich é amplamente utilizado, sendo expresso por:

$$
\mathrm{t}_{\mathrm{c}}=0,95 \cdot\left(\frac{\mathrm{L}^{3}}{\mathrm{H}}\right)^{0,385}
$$

onde tc é o tempo de concentração (h), L é o comprimento do talvegue $(\mathrm{km})$ e $\mathrm{H}$ é o desnível médio do talvegue $(\mathrm{m})$.

\section{Tempo de retorno}

O tempo de retorno ou recorrência (Tr) é definido como o inverso da probabilidade de um determinado evento hidrológico ser igualado ou superado em um ano qualquer. A probabilidade de este evento ocorrer em um ano qualquer é definida por:

$$
\mathrm{T}=\frac{1}{\mathrm{~F}}
$$

onde F é a probabilidade de ocorrência do evento, ou frequência, e $\mathrm{T}$ é o tempo de retorno ou recorrência.

\section{Determinação da equação de chuvas intensas}

As equações de chuvas intensas podem ser expressas por equações genéricas que têm a seguinte forma:

$$
i=\frac{a T^{b}}{(t+c)^{d}}
$$

onde $\mathrm{i}=$ intensidade de precipitação, em $\mathrm{mm} / \mathrm{h} ; \mathrm{T}$ $=$ tempo de retorno da precipitação, em anos; $\mathrm{t}=$ duração da precipitação, em minutos; e a,b,c e d = parâmetros de ajuste estatístico.

Optou-se por utilizar a metodologia de Bell (1969) e o método das relações entre durações (CETESB, 1980) para obtenção dos parâmetros das chuvas intensas (a-d) da bacia do Riacho da Cachoeira.

Segundo Righetto (1998), o método de Bell associa a altura pluviométrica de uma chuva intensa de duração dc e período de retorno $\mathrm{T}$, isto é, $\mathrm{h}(\mathrm{dc} ; \mathrm{T})$, com uma chuva intensa padrão de 60 minutos de duração e 2 anos de período de retorno, $\mathrm{h}(60 ; 2)$, utilizando-se a seguinte relação empírica:

$\mathrm{h}_{\left(\mathrm{d}_{\mathrm{c}} ; \mathrm{T}\right)}=\left(\mathrm{a}_{0} \cdot \ln \mathrm{T}+\mathrm{a}_{1}\right) \cdot\left(\mathrm{a}_{2} \cdot \mathrm{d}_{\mathrm{c}}^{\mathrm{b}}-\mathrm{a}_{3}\right) \cdot \mathrm{h}_{(60 ; 2)}$ Eq. (4)

onde $a_{0}, a_{1}, a_{2}, a_{3}$ e b são parâmetros regionais. A utilização de dados de postos brasileiros levou à fixação de valores para esses parâmetros, de modo que $\mathrm{h}(\mathrm{dc} ; \mathrm{T})$ pode ser expresso por:

$\mathrm{h}_{\left(\mathrm{d}_{\mathrm{c}} ; \mathrm{T}\right)}=(0,31 \cdot \ln \mathrm{T}+0,7) \cdot\left(0,38 \cdot \mathrm{d}_{\mathrm{c}}{ }^{0,31}-0,39\right) \cdot \mathrm{h}_{(60 ; 2)}$

Como o método só se aplica se for possível estimar h $(60 ; 2)$, recomenda-se que seja utilizada a seguinte relação empírica (Coutinho et al., 2013):

$$
\mathrm{h}_{(60 ; 2)}=\mathrm{K} \cdot \mathrm{h}_{\mathrm{dia}(2)}
$$

onde $\mathrm{K}$ é igual a 0,510 e hdia(2) é a altura pluviométrica máxima diária anual correspondente ao período de retorno $\mathrm{T}=2$ anos.

$\mathrm{O}$ método da desagregação de chuvas desenvolvido pelo CETESB (1980) adota o fator médio de 1,14 para a transformação de chuva máxima de 1 dia em chuva de 24 horas; para reduzir a chuva de 24 horas em chuvas de $12 \mathrm{~h}$, $10 \mathrm{~h}, 8 \mathrm{~h}, 6 \mathrm{~h}$ e $1 \mathrm{~h}$ são utilizados os fatores 0,85 ; 0,$82 ; 0,78 ; 0,72$ e 0,42 . A chuva de 30 minutos é obtida multiplicando-se a chuva de 1 hora por 0,74; e para obter as chuvas de $25 \mathrm{~min}, 20 \mathrm{~min}, 15$ min, $10 \mathrm{~min}$ e $5 \mathrm{~min}$, multiplica-se a chuva de 30 min por 0,$91 ; 0,81 ; 0,70 ; 0,54$ e 0,34 respectivamente.

Para a determinação da chuva de 1 dia, utilizou-se a distribuição de variáveis aleatórias de Gumbel, que pode ser expressa em sua forma inversa por:

$$
\mathrm{x}(\mathrm{T})=\beta-\alpha \ln \left(-\ln \left(1-\frac{1}{\mathrm{~T}}\right)\right)
$$

onde $\beta$ o parâmetro de posição, $\alpha$ o parâmetro de escala, e $\mathrm{T}$ o tempo de retorno em anos. Estimando os parâmetros da distribuição pelo método dos momentos (Naghettini \& Pinto, 2007) obtém-se:

$$
\begin{gathered}
\beta=x-0,45 S \\
\alpha=S / 1,283
\end{gathered}
$$


onde x e S representam, respectivamente, a média e o desvio padrão amostrais.

Para avaliar a qualidade dos ajustes da distribuição estatística foi utilizado o teste de aderência de Kolmogorov-Smirnov (KS), com nível de significância de 5\%. Este é um teste não paramétrico, cuja estatística de teste tem como base a diferença máxima entre as funções de probabilidades acumuladas, empírica e teórica. A estatística do teste KS é dada por:

$$
\mathrm{D}_{\mathrm{N}}=\sup _{-\infty<\mathrm{x}<\infty}\left|\mathrm{F}_{\mathrm{N}}(\mathrm{x})-\mathrm{F}(\mathrm{x})\right|
$$

Corresponde, portanto, à maior diferença entre as probabilidades empírica $\left(\mathrm{F}_{\mathrm{N}}(\mathrm{x})\right)$ e teórica $(\mathrm{F}(\mathrm{x}))$.

\section{Hietogramas de projeto}

Para a construção dos hietogramas de projeto, foram utilizados o método do Bureau of Reclamation e o método dos Blocos Alternados. Essas metodologias distribuem a precipitação ao longo do tempo de forma a buscar um cenário crítico de precipitação.

As duas metodologias propõem a desagregação dos totais de chuva em intervalos de tempo discretizados pela sua duração total, ou tempo de concentração. Desta forma, discretiza-se o tempo de concentração em intervalos de tempo iguais e determina-se as alturas de chuvas correspondentes às diversas durações cumulativas por meio da equação IDF. Em seguida, obtém-se os incrementos de alturas de chuva correspondentes a cada incremento de duração. Estes incrementos são então rearranjados de modo a obter hidrogramas com elevados picos.

$\mathrm{O}$ método do Bureau of Reclamation sugere que os incrementos sejam rearranjados na seguinte ordem de magnitude: $6,4,3,1,2$ e 5, segundo Tucci (2009). O método dos Blocos Alternados reordena os incrementos de modo que o maior valor se localize no centro da duração total da chuva, sendo os demais dispostos em ordem decrescente, à direita e à esquerda do bloco central, alternadamente (Tucci, 1995).

\section{Método do SCS}

O método foi desenvolvido pelo National Resources Conservation Center dos Estados Unidos e admite que, na sequência de uma precipitação intensa, a razão entre a altura de precipitação retida na bacia hidrográfica após o início do escoamento superficial e a capacidade máxima de retenção de água na bacia é igual à razão entre a precipitação efetiva e o escoamento superficial potencial. $\mathrm{O}$ escoamento superficial potencial é definido como sendo a precipitação deduzida das perdas iniciais que ocorrem até a saturação da superfície. Assim, obtém-se:

$$
\frac{F_{a}}{S}=\frac{P_{e}}{P-I_{a}}
$$

onde $\mathrm{P}$ é a precipitação $(\mathrm{mm}), \mathrm{P}_{\mathrm{e}}$ é a precipitação efetiva $(\mathrm{mm}), \mathrm{F}_{\mathrm{a}}$ é a altura de precipitação retida na bacia hidrográfica após o início do escoamento superficial, representando, portanto, as perdas contínuas de precipitação $(\mathrm{mm}), \mathrm{S}$ é a retenção potencial máxima $(\mathrm{mm}) ; \mathrm{I}_{\mathrm{a}}$ são as perdas iniciais, compreendendo todas as perdas por interceptação, armazenamento em depressões, evapotranspiração e infiltração que antecedem a saturação da superfície e, consequentemente, a formação do escoamento à superfície do terreno $(\mathrm{mm})$, e $\mathrm{P}-\mathrm{I}_{\mathrm{a}}$ é o escoamento superficial potencial (mm).

Assumindo a hipótese que a altura de precipitação retida na bacia hidrográfica após o início do escoamento superficial é a diferença entre a precipitação e a precipitação efetiva $\left(\mathrm{F}_{\mathrm{a}}=\right.$ $\mathrm{P}-\mathrm{P}_{\mathrm{e}}$ ). O SCS admite que as perdas iniciais correspondam a $20 \%$ da capacidade máxima de retenção de água na bacia hidrográfica. Assim, a expressão para o cálculo da precipitação efetiva do evento é dada por:

$$
P_{e}=\frac{(P-0,2 \cdot S)^{2}}{(P+0,8 . S)}
$$

A equação 7 é válida apenas para $\mathrm{P}>$ $0,2 . \mathrm{S}$, caso contrário, $\mathrm{Pe}=0$. Para determinar a retenção máxima da camada superior do solo $\mathrm{S}$, foi relacionado tal parâmetro da bacia com um fator "Curva Número" CN pela seguinte expressão:

$$
\mathrm{S}=\frac{25400}{\mathrm{CN}}-254
$$

O parâmetro $\mathrm{CN}$ varia em uma escala de 1 a 100, que retrata as condições de cobertura e solo, variando de uma cobertura muito permeável (limite inferior) a uma cobertura completamente impermeável (limite superior).

$\mathrm{Na}$ Tabela 2 são apresentados os valores típicos do parâmetro CN (Tucci, 2009). A sua determinação é função do uso do solo na bacia e do grupo de solo conforme as características descritas na Tabela 3. 
Tabela 2. Número de Curva $(\mathrm{CN})$ para diferentes condições do complexo hidrológico. Fonte: Adaptado de Tucci (2009).

\begin{tabular}{|c|c|c|c|c|c|}
\hline Uso do solo & Superfície & A & B & $\mathbf{C}$ & $\mathbf{D}$ \\
\hline \multirow{3}{*}{ Pastagens } & Pobres, em curvas de nível. & 47 & 67 & 81 & 88 \\
\hline & Normais, em curvas de nível. & 25 & 59 & 75 & 83 \\
\hline & Boas, em curvas de nível. & 6 & 35 & 70 & 79 \\
\hline \multirow{4}{*}{ Campos permanentes } & Normais & 30 & 58 & 71 & 78 \\
\hline & Esparsas, de baixa transpiração. & 45 & 66 & 77 & 83 \\
\hline & Normais & 36 & 60 & 73 & 79 \\
\hline & Densas, de alta transpiração. & 25 & 55 & 70 & 77 \\
\hline \multirow{3}{*}{ Estradas de terra } & Normais & 56 & 75 & 86 & 91 \\
\hline & Más & 72 & 82 & 87 & 89 \\
\hline & Superfície dura & 74 & 84 & 90 & 92 \\
\hline \multirow{4}{*}{ Florestas } & Muito esparsas, baixa transpiração. & 56 & 75 & 86 & 91 \\
\hline & Esparsas & 46 & 68 & 78 & 84 \\
\hline & Densas, alta transpiração. & 26 & 52 & 62 & 69 \\
\hline & Normais & 36 & 60 & 70 & 76 \\
\hline
\end{tabular}

Tabela 3. Grupos de Solo, segundo Manual de Hidrologia Básica para Estruturas de Drenagem. Fonte: Adaptado de DNIT (2005).

\begin{tabular}{cc}
\hline SOLO A & Solos que produzem baixo escoamento superficial e alta infiltração. \\
SOLO B & $\begin{array}{l}\text { Solos menos permeáveis do que o anterior, solos arenosos menos profundo do que o tipo A e } \\
\text { com permeabilidade superior à média. }\end{array}$ \\
SOLO C & $\begin{array}{c}\text { Solos que geram escoamento superficial acima da média e com capacidade de infiltração } \\
\text { abaixo da média, contendo percentagem considerável de argila e pouco profundo. }\end{array}$ \\
SOLO D & $\begin{array}{r}\text { Solos contendo argilas expansivas e pouco profundas com muito baixa capacidade de } \\
\text { infiltração, gerando a maior proporção de escoamento superficial. }\end{array}$ \\
\hline
\end{tabular}

\section{Hidrograma unitário}

Para transformar a precipitação de projeto em vazão na bacia, é necessário aplicar um modelo de transformação chuva-vazão. O método do hidrograma unitário (HU) representa a resposta da bacia a uma precipitação de volume unitário de duração $\Delta \mathrm{t}$.

O hidrograma unitário pode ser determinado com base nas características físicas da bacia hidrográfica. Na Figura 2 podem ser visualizados os parâmetros que caracterizam o hidrograma unitário triangular do SCS.

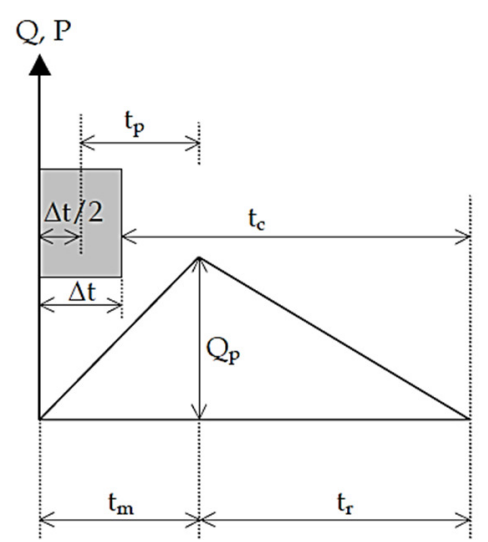

Figura 2. Hidrograma unitário triangular do SCS.
Os parâmetros do hidrograma unitário triangular do SCS são determinados da seguinte forma:

1. Determinar o tempo de concentração $\left(t_{c}\right)$ da bacia.

2. Determinar o parâmetro $\mathrm{t}_{\mathrm{m}}($ Equação 9$)$ :

$$
\mathrm{t}_{\mathrm{m}}=\frac{\Delta \mathrm{t}}{2}+0,6 \cdot \mathrm{t}_{\mathrm{c}}
$$

onde $\Delta \mathrm{t}$ é o intervalo de tempo de simulação, obtido a partir da precipitação, e t $\mathrm{c}_{c}$ e o tempo de concentração da bacia.

3. Determinar o tempo de pico do hidrograma, $\mathrm{t}_{\mathrm{p}}$ :

$$
t_{p}=0,6 \cdot t_{c}
$$

4. Determinar o tempo de recessão do hidrograma, $t_{r}$ :

$$
t_{r}=1,67 \cdot t_{p}
$$

5. Determinar o tempo de base do hidrograma, $\mathrm{t}_{\mathrm{b}}$ :

$$
\mathrm{t}_{\mathrm{b}}=\mathrm{t}_{\mathrm{m}}+\mathrm{t}_{\mathrm{r}}
$$


6. Determinar a vazão máxima utilizando a equação 10 :

$$
\mathrm{Q}_{\mathrm{p}}=\frac{0,208 \cdot \mathrm{A}}{\mathrm{t}_{\mathrm{m}}}
$$

onde $\mathrm{Q}_{\mathrm{p}}$ é a vazão máxima do hidrograma triangular $\left(\mathrm{m}^{3} / \mathrm{s} / \mathrm{mm}\right)$ e A é a área da bacia $\left(\mathrm{km}^{2}\right)$.

$O$ intervalo de tempo é definido em unidades de $\mathrm{t}_{\mathrm{p}}$. Recomenda-se a utilização de $\Delta \mathrm{t}=$ $\mathrm{t}_{\mathrm{p}} / 5$. Para cada intervalo de tempo, multiplica-se a precipitação pelas ordenadas do HU. O hidrograma de projeto é obtido somando-se os hidrogramas individuais de cada intervalo de precipitação.

\section{Resultados}

$\mathrm{Na}$ região semiárida do nordeste brasileiro, os solos podem ser classificados, na sua maioria, como rasos, apresentando baixa capacidade de infiltração. Como a bacia do Riacho da Cachoeira encontra-se inserida neste contexto, foi considerado que o solo da bacia é predominantemente do grupo C (Tabela 2). Considerando o uso do solo (Tabela 3), a área da bacia é ocupada basicamente por vegetação típica da Caatinga, podendo ser classificado como uma floresta de superfície normal. De acordo com essas características, a bacia do riacho da Cachoeira apresenta $\mathrm{CN}=70$.

$\mathrm{Na}$ Tabela 4 são apresentadas as características físicas da bacia do riacho da Cachoeira, assim como o valor do tempo de concentração determinado através da equação de Kirpich (equação 1).

Tabela 4. Características físicas da bacia e tempo de concentração.

\begin{tabular}{lc}
\hline Área $\left(\mathbf{k m}^{2}\right)$ & 171,17 \\
$\mathbf{L}(\mathbf{k m})$ & 27,557 \\
Hmon $(\mathbf{m})$ & 820,00 \\
Hjus (m) & 428,00 \\
S (m.m & -1 \\
tc $(\mathbf{m i n})$ & 0,01423 \\
\hline
\end{tabular}

A distribuição teórica obtida por Gumbel, bem como a distribuição empírica dos dados de precipitação máxima diária anual estão apresentadas na Figura 3. A diferença máxima obtida entre a distribuição empírica e a teórica foi de 0,14 , enquanto a diferença crítica permitida para a quantidade de dados observados, ao nível de significância de $5 \%$, é de 0,19 , portanto, a distribuição Gumbel foi capaz de representar as precipitações extremas na região. Para a utilização do método de Bell (1969) - Equação 4, obteve-se, a partir da Figura 3, a intensidade de chuva diária máxima anual para o tempo de retorno de 2 anos $(76,2 \mathrm{~mm})$, e a partir da multiplicação desta por um coeficiente de desagregação $(\mathrm{k}=0,51)$ foi possível obter o parâmetro de chuva de duração de 60 minutos e tempo de retorno de dois anos $(38,86$ $\mathrm{mm})$.

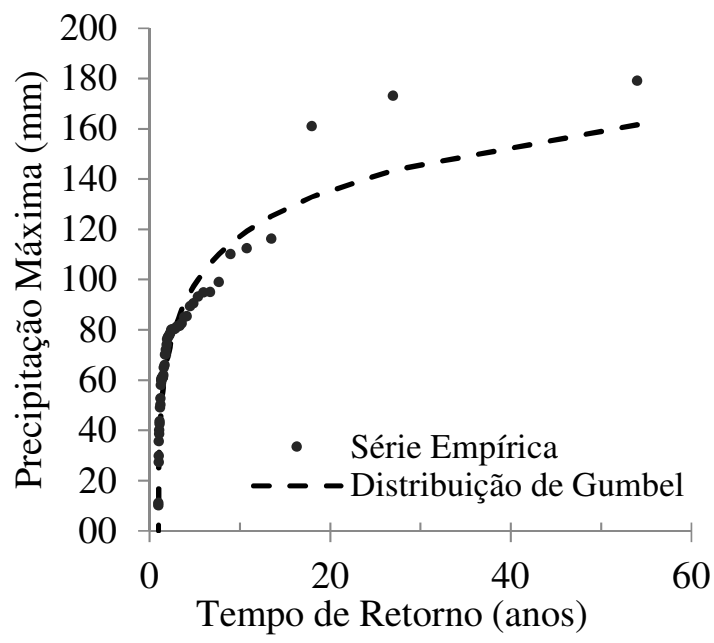

Figura 3. Precipitações máximas diárias anuais retiradas da série pluviométrica para cada Tempo de Retorno correspondente e distribuição teórica estimada por Gumbel. Fonte: Adaptado ANA (2012).

$\mathrm{Na}$ Figura 4 são demonstrados os parâmetros das equações de chuvas intensas, determinados pelos métodos de Bell e de Relações entre durações. O método de Bell apresentou valores de 472,$88 ; 0,20 ; 7,30$; e 0,64 para os parâmetros a, b, c e d, respectivamente. O método das relações entre durações apresentou valores de 853,$72 ; 0,21 ; 11,83$; e 0,77 para os parâmetros a, b, c e d, respectivamente. O método das relações entre durações apresentou um coeficiente de determinação de $99,79 \%$, enquanto o método de Bell mostrou um coeficiente de 99,64\%.

Na Figura 5 é apresentado o hidrograma unitário da bacia do Riacho da Cachoeira. O hidrograma unitário apresentou uma vazão de pico de $13,3 \mathrm{~m}^{3} \cdot \mathrm{s}^{-1} \cdot \mathrm{mm}^{-1}$, no tempo de 2,7 horas.

Os hidrogramas determinados para cada método de desagregação de chuva e para cada chuva de projeto obtida estão apresentados na Figura 6. Foram calculados hidrogramas para os tempos de retorno de 2, 10, 25, 50, 100 anos. Para esses hidrogramas, a vazão máxima obtida por eles e a precipitação efetiva que ocasionou esse escoamento estão apresentadas na Tabela 5. 

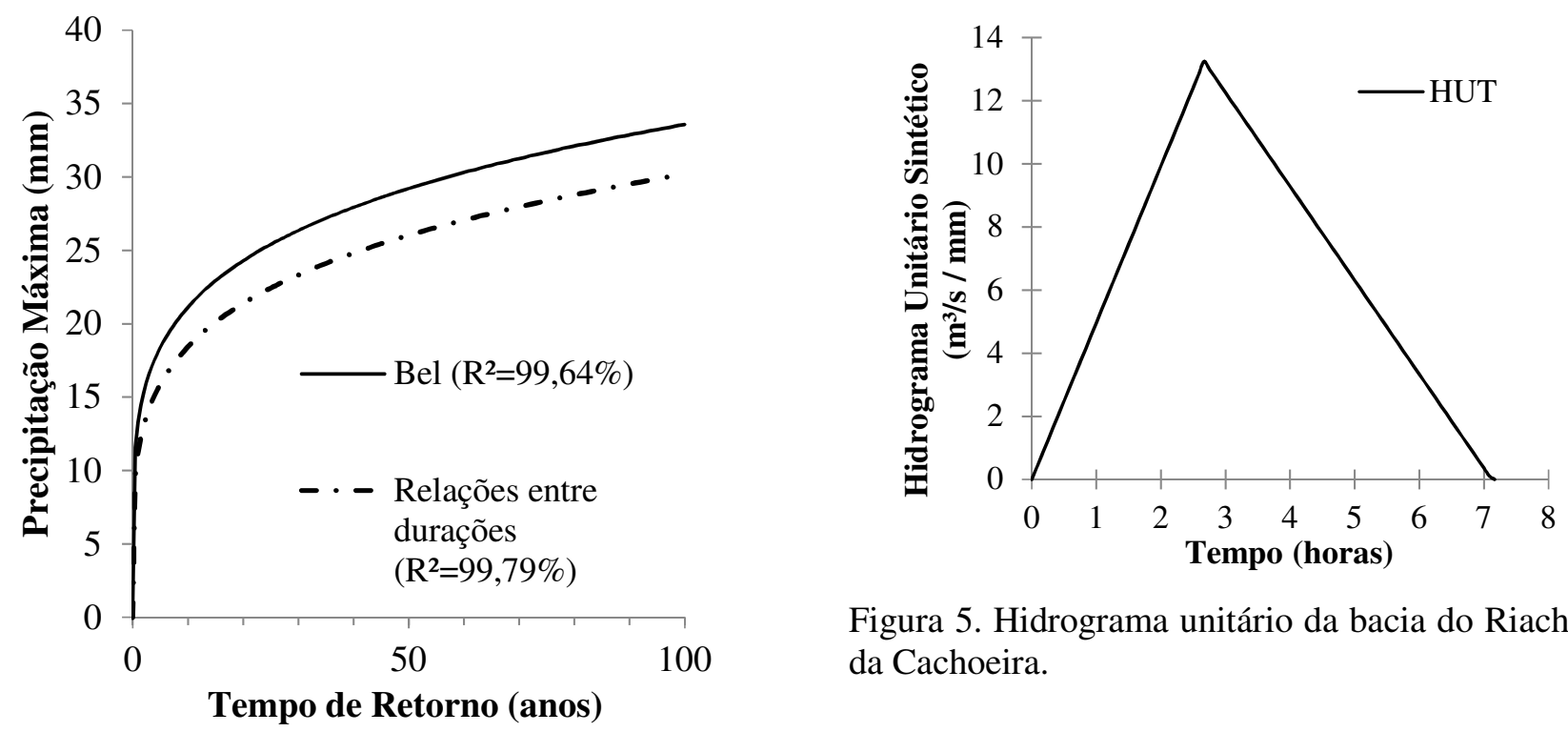

Figura 5. Hidrograma unitário da bacia do Riacho da Cachoeira.

Figura 4. Comparação entre as IDFs geradas a partir da equação de Bell (1969) e do método das relações entre durações.

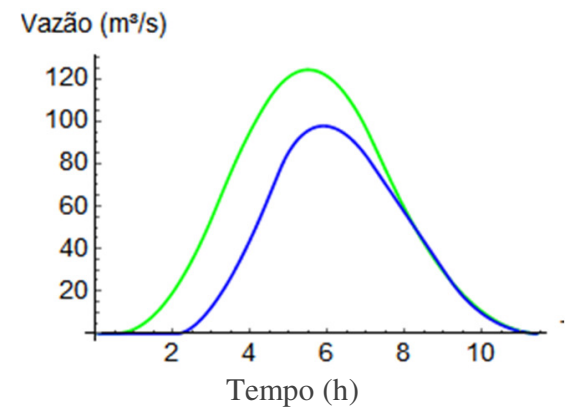

(A.1)

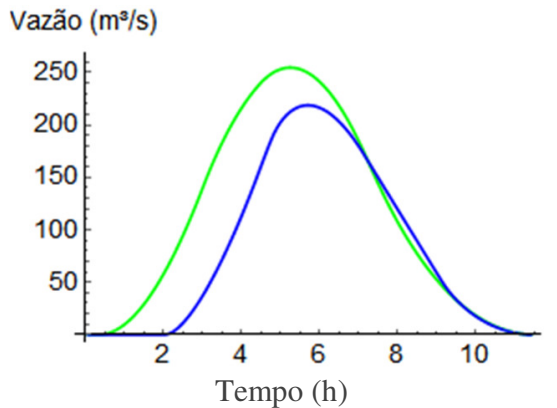

(A.2)

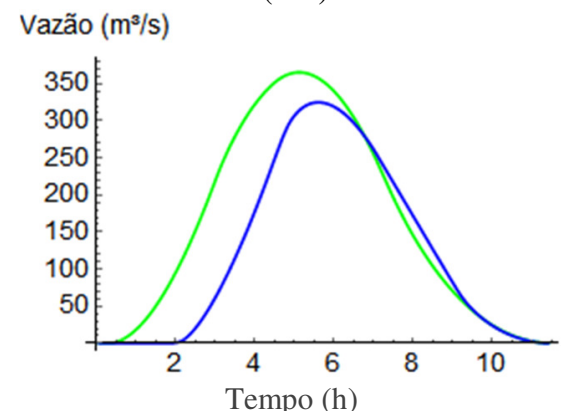

(A.3)

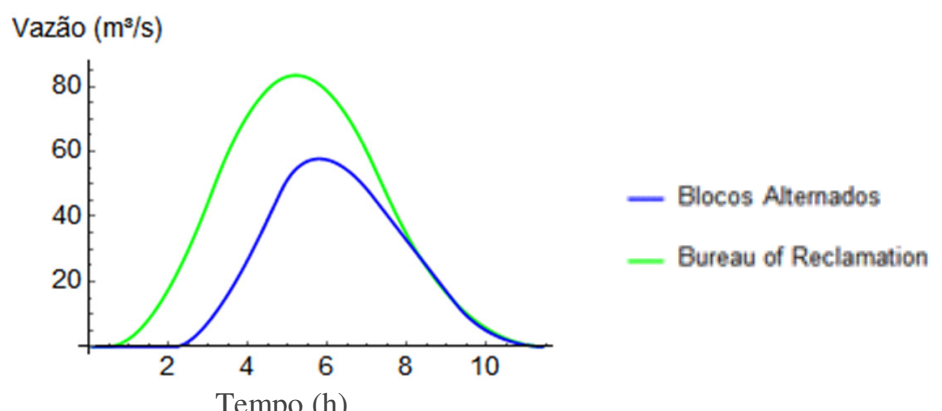

(B.1)

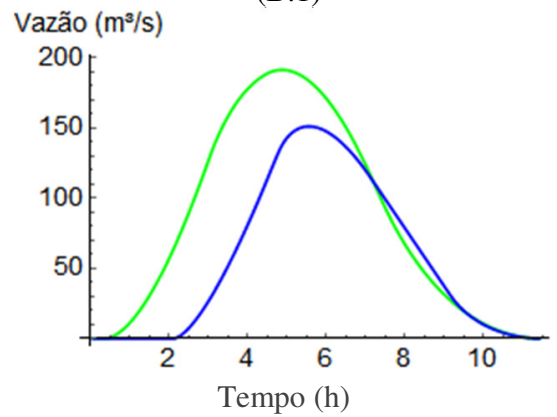

(B.2)

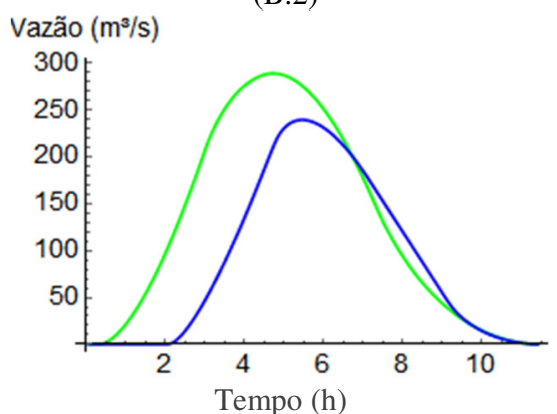

(B.3) 


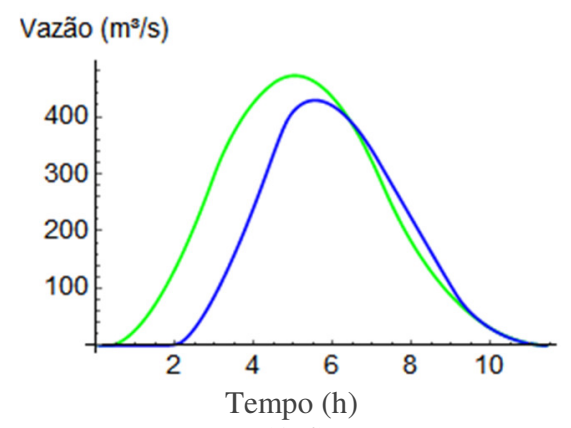

(A.4)

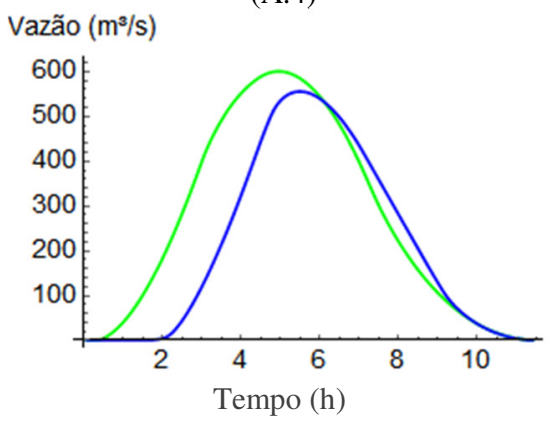

(A.5)

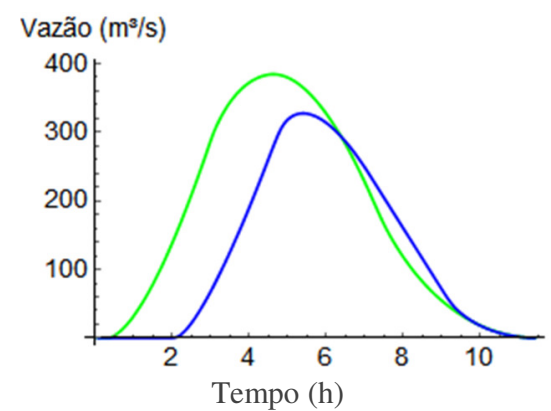

(B.4)

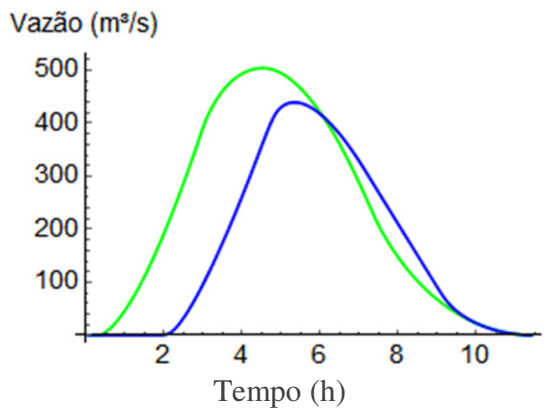

(B.5)
- Blocos Alternados

- Bureau of Reclamation

Figura 6. Hidrogramas estimados a partir do método de Bell (A) e relações entre durações (B) para os tempos de retorno de: 1) 2 anos; 2) 10 anos; 3) 25 anos; 4) 50 anos; e 5) 100 anos;

Tabela 5. Vazões de pico obtidas nos hidrogramas e suas respectivas precipitações efetivas.

\begin{tabular}{|c|c|c|c|c|c|}
\hline \multirow[b]{2}{*}{$\begin{array}{c}\text { Grandeza } \\
\text { hidrológica }\end{array}$} & \multirow{2}{*}{$\begin{array}{c}\text { Tempo } \\
\text { de } \\
\text { retorno } \\
\text { (anos) }\end{array}$} & \multicolumn{2}{|c|}{ Relações entre durações } & \multicolumn{2}{|c|}{ Bell } \\
\hline & & $\begin{array}{c}\text { Bureau of } \\
\text { Reclamation }\end{array}$ & $\begin{array}{c}\text { Blocos } \\
\text { Alternados }\end{array}$ & $\begin{array}{c}\text { Bureau of } \\
\text { Reclamation }\end{array}$ & $\begin{array}{c}\text { Blocos } \\
\text { Alternados }\end{array}$ \\
\hline \multirow{5}{*}{ 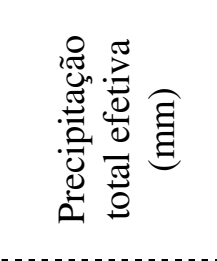 } & 2 & 8,80 & 5,22 & 12,93 & 8,92 \\
\hline & 10 & 20,59 & 13,68 & 27,22 & 20,08 \\
\hline & 25 & 31,14 & 21,57 & 39,43 & 29,87 \\
\hline & 50 & 41,52 & 29,50 & 51,16 & 39,41 \\
\hline & 100 & 54,40 & 39,48 & 65,44 & 51,13 \\
\hline \multirow{5}{*}{ 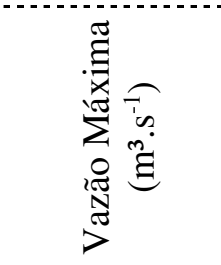 } & 2 & 83,35 & 57,73 & 124,21 & 97,76 \\
\hline & 10 & 191,64 & 151,39 & 254,70 & 218,75 \\
\hline & 25 & 288,73 & 239,16 & 365,35 & 324,98 \\
\hline & 50 & 384,70 & 327,80 & 471,58 & 428,57 \\
\hline & 100 & 504,20 & 439,56 & 600,84 & 555,98 \\
\hline
\end{tabular}

\section{Discussão}

Os parâmetros de escala $(\alpha=25,67)$ e posição $(\beta=59,44)$ obtidos pela distribuição de Gumbel estão em conformidade com os obtidos por Coutinho et al. (2013): $\alpha$ entre 15,50 e 60,34, e $\beta$ entre 18,42 e 100,20. Eles também estão dentro do intervalo obtido por Back (2001) em Santa Catarina ( $\alpha$ entre 15 e 36 e $\beta$ entre 57 e 115) e Caldeira et al. (2015) no Rio Grande do Sul $(\alpha$ entre 7 e 50 e $\beta$ entre 47 e 110). Para um tempo de retorno maior que 15 anos, a distribuição teórica apresentou um subestimativa em relação aos dados empíricos.

Em relação às duas equações de chuvas intensas obtidas, no tempo de concentração da bacia o método das relações entre durações gera intensidades pluviométricas menores que o método de Bell. Os parâmetros a e b estimados por Bell estão dentro do intervalo obtido para Pernambuco por Coutinho et al. (2013): a entre 265,97 e 707,86, e b entre 0,18 e 0,21 , entretanto o parâmetro a do método das relações entre durações, mostrou-se maior que este intervalo. Os parâmetros c e d obtidos pelos dois métodos foram maiores que os determinados por Coutinho et al. (2013): c entre 2,62 e 2,83, e b igual a 0,55. Os parâmetros de chuvas intensas obtidos pelo método das relações entre durações também estão entre os valores obtidos por Silva \& Oliveira (2017) para o nordeste brasileiro: a) entre 280,62 e 1669,11, b) entre 0,03 e 0,30, c) entre 8,93 e 
13,11, e d) entre 0,7 e 0,79. Considerando Bell, os parâmetros $\mathrm{c}$ e d foram menores.

Segundo Garcia et al. (2011), os parâmetros das equações intensidade-duraçãofrequência (IDF) apresentam alta variabilidade independente do método, no entanto o método da Relação entre durações apresentou melhor desempenho global em comparação com o método de Bell no estado do Mato Grosso, isso também ocorreu nesse estudo e pode ser percebido por meio do coeficiente de determinação.

A vazão máxima gerada por todos os modelos é cerca de $10 \mathrm{~m}^{3} \cdot \mathrm{s}^{-1} \cdot \mathrm{mm}^{-1}$ da precipitação efetivamente escoada. $\mathrm{O}$ hietograma obtido pelo método do Bureau of Reclamation gerou uma precipitação maior que o obtido pelo método dos Blocos Alternados, como também os seus picos de vazão, essa divergência diminui com o aumento do tempo de retorno da precipitação. Assim como as intensidades de chuva, o método de Bell gerou vazões maiores que o método das relações entre durações.

\section{Conclusão}

A distribuição de Gumbel demonstrou ser apropriada para retratar as estimativas dos valores das chuvas máxima com grau de significância de 5\% pelo método de Kolmogorov-Smirnov. Os dois métodos de desagregação de chuvas apresentaram bons ajustes, com coeficientes de determinação superiores a $99 \%$.

As IDFs geradas, comparadas com a duração do tempo de concentração, não apresentaram grande divergência entre si.

Os hidrogramas obtidos apresentaram divergências entre si, tendo o método de Bell, com a chuva de projeto obtida pelo Bureau of Reclamation, apresentado as maiores vazões de pico e o método das relações entre durações, com a chuva determinada a partir do método dos blocos alternados, apresentado as menores magnitudes de vazões máximas.

\section{Agradecimentos}

O estudo foi realizado com apoio do Conselho Nacional de Desenvolvimento Científico e Tecnológico (CNPq), processo $\mathrm{N}^{\circ}$ 465764/2014-2, da Coordenação de Aperfeiçoamento de Pessoal de Nível Superior Brasil (CAPES), Processo $\mathrm{N}^{\circ}$ 88887,136369/2017-00 e da Fundação de Amparo à Ciência e Tecnologia de Pernambuco (FACEPE), Processo: APQ-0498-3,07/17, através do projeto "Observatório Nacional da Dinâmica da Água e de Carbono no Bioma Caatinga (ONDACBC)".

\section{Referências}

ANA. Agência nacional de Águas e Climas, Hidroweb. Disponível em: http://hidroweb.ana.gov.br. Acesso em: 01/03/2012.

BACK, A. J. 2001. Seleção de distribuição de probabilidade para chuvas diárias extremas do Estado de Santa Catarina. 2001. Revista Brasileira de Meteorologia, v. 14, n. 1, p. 59-72.

BELL, F. C. 1969. Generalized rainfall-durationfrequency relationships, Journal of the Hydraulics, Division-ASCE, v. 95, p. 311-327.

BEMFICA, D. C.; GOLDENFUM, J. A.; SILVEIRA, A. L. L. 2000. Análise da Aplicabilidade de padrões de chuva de projeto a Porto Alegre. Revista Brasileira de Recursos Hídricos, v. 5, n. 5, p. 5-16.

CALDEIRA, L. T.; BESKOW, S.; MELLO, C. R., FARIAS, L. C.; SOUZA, M. R.; GUEDES, H. A. S. 2015. Modelagem probabilística de eventos de precipitação extrema no estado do Rio Grande do Sul. Revista Brasileira de Engenharia Agrícola e Ambiental, v. 19, n. 3, p. 197-203.

CARVALHO, M. A.; CHAUDRHY, F. H. 2001. Aplicação de Hidrograma Unitário Geomorfológico na Previsão de Respostas Hidrológicas. Revista Brasileira de Recursos Hídricos, v. 6, n. 4, p. 5-17.

CETESB. 1980. Drenagem urbana: Manual de projeto. São Paulo, 466p.

COUTINHO, A. P.; RIBAS, L. V.; LEITE, L. L. L.; ANTONINO, A. C. D.; CABRAL, J. J. S. P.; MONTENEGRO, S. M. G. L.; MELO, T. A. T. 2013. Determinação de equações de chuvas intensas para municípios das mesorregiões do estado de Pernambuco através do método de Bell. XX Simpósio brasileiro de Recursos Hídricos, Bento Gonçalves/RS, pp. 1-8.

CUNHA, S. F. C.; OLIVEIRA E SILVA; F. E. MOTA; TAINÁ, ULHPA; PINHEIRO, M. C. 2015. Avaliação da acurácia dos métodos do SCS para cálculo da precipitação efetiva e hidrogramas de cheia. Revista Brasileira de Recursos Hídricos, v. 20, n. 4 , p. $837-848$.

DAMÉ, R. C. F.; TEIXEIRA, C. F. A.; TERRA, V. S. S.; ROSSOKOFF, J. L. C. 2010. Hidrograma de projeto em função da metodologia 
utilizada na obtenção da precipitação. Revista Brasileira de Engenharia Agrícola e Ambiental, v. 14, n. 1, p. 46-54.

DNIT. 2005. Departamento Nacional de InfraEstrutura de Transportes. Manual de hidrologia básica para estrutura de drenagem, 2a ed,, Rio de Janeiro, 133p.

DRUMOND, P. P.; MOURA, P. M.; COELHO, M. M. L. P. 2018. Comparison the monitoring data of an on-site stormwater detention (OSD) and the results in the use of theoretical methods for its design. RBRH, v. 23, n. 18, p. 1-12.

GARCIA, S. S.; AMORIM, R. S. S.; COUTO, E. G.; STOPA, W. H. 2011. Determinação da equação intensidade-duração-frequência para três estações meteorológicas do Estado de Mato Grosso. Revista Brasileira de Engenharia Agricola e Ambiental, v. 15, n.6, p. 575+.

MATA-LIMA, H.; VARGAS, H.; CARVALHO, J.; GONÇALVES, M.; CAETANO, H.; MARQUES, A.; RAMINHOS, C. 2007. Comportamento hidrológico de bacias hidrográficas: integração de métodos e aplicação a um estudo de caso Rem: Revista Escola de Minas, v. 60 , n. 3 , p. 525-536.

NAGHETTINI, M.; PINTO, E. J. DE A. 2007. Hidrologia Estatística. CPRM. 600p.
NUNES, F. G.; FIORI, A. P. 2007. A utilização do método de Ven Te Chow -Soil Conservation Service (SCS) na estimativa da vazão máxima da bacia hidrográfica do rio Atuba. Revista Eletrônica Geografar, v.2, n.2, p.139-155.

PORTELA, M. M.; MARQUES, P.; CARVALHO, F. F. 2000. Hietogramas de projecto para a análise de cheias baseada no modelo do hidrograma unitário do Soil Conservation Service (SCS). $5^{\circ}$ Congresso da Água - A Água e o Desenvolvimento Sustentável: Desafios para o Novo Século. pp. 1-17.

RIGHETTO, A. M. 1998. Hidrologia e Recursos Hídricos. EESC/USP, São Carlos-SP, 840 p.

SILVA, C. B; OLIVEIRA, L. F. C. 2017. Relação intensidade-duração-frequência de chuvas extremas na região nordeste do Brasil. Revista Brasileira de Climatologia, v. 20, p. 267-283.

TUCCI, C. E. M. 1995. Inundações urbanas. In: TUCCI, C. E. M; PORTO, R. L. L.; BARROS, M. T. Drenagem urbana: Porto Alegre: Editora da Universidade/UFRGS/ABRH. 1 ed. Cap. 1, pp. 15-36.

TUCCI, C. E. M. 2009. Hidrologia Ciência e Aplicação. Editora da UFRGS, Porto Alegre, 943p. 